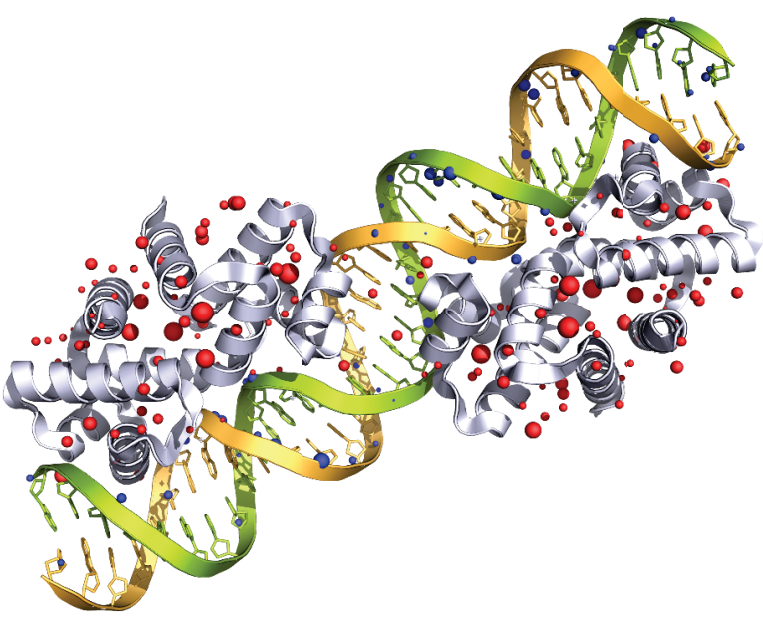

Figure 1. Representation of specific damage distribution throughout the C.Esp1396I complex at 44.63 MGy (1). Specific damage sites are represented as spheres, with radii proportional to electron density loss. Spheres closer/ further than $2 \AA$ to/from the DNA strands are coloured blue/red.

Keywords: macromolecular X-ray crystallography, radiation damage, protein-nucleic acid complexes

\section{MS7-P10 Structural studies on DNA cleavage-and-ligation nucleases of mobile genetic elements involved in spread of antibiotic resistance}

Radoslaw Pluta ${ }^{1,2}$, D Roeland Boer ${ }^{1}$, Alicia Guasch ${ }^{1}$, Silvia Russi $^{1}$, José Ruiz-Maso ${ }^{3}$, Cris Fernandez-Lopez ${ }^{3}$, Fabian Lorenzo-Diaz ${ }^{3}$, Maria Lucas ${ }^{4}$, Gloria del Solar ${ }^{3}$, Fernando de la $\mathrm{Cruz}^{4}$, Manuel Espinosa ${ }^{3}$, Miquel Coll ${ }^{1}$

1. Institute for Research in Biomedicine (IRB Barcelona), Spain and Institut de Biologia Molecular de Barcelona (CSIC), Spain 2. International Institute of Molecular and Cell Biology in Warsaw (IIMCB Warsaw), Poland

3. Centro de Investigaciones Biológicas (CSIC), Madrid, Spain 4. Instituto de Biomedicina y Biotecnologia de Cantabria (IBBTEC), Santander, Spain

email: rpluta@genesilico.pl

Plasmids and integrative and conjugative elements (ICEs) are major mobile genetic elements (MGEs) that provide routes for rapid acquisition of new genetic information in bacteria and therefore contribute to the spread of antibiotics resistance. Essential for their action are plasmid/ICE-encoded site- and strand-specific one-metal-ion endonucleases called relaxases. Conjugative relaxases cleave a single strand of the DNA substrate by formation of an intermediate covalent adduct with the scissile phosphate of the DNA nic site. After the ssDNA-relaxase molecule is transferred to the recipient cell, relaxases ensure re-ligation of their DNA cargo. Additionally, plasmids and some ICEs encode for DNA replication relaxases, crucial for their maintenance. Understanding plasmid/ICEs conjugal transfer and replication may aid in combating the spread of antibiotics resistance as well as contribute to the development of new tools for DNA delivery into human cells. Structures of replicative and conjugative relaxases RepB, MobM and TrwC that were solved in our lab are compared herein.

Keywords: relaxases, endonucleases, bacterial conjugation, plasmid replication, antibiotic resistance 